\title{
Effect of Nanosilver and Antibiotics Separately and in Combination on Pseudomonas aeruginosa Clinical Isolates
}

\author{
Alyousef Mansour Ali ${ }^{1 *}$, Abdulaziz Ibrahim Alsenaid ${ }^{2}$, Ahmed Hassan Al-Mosilhi ${ }^{3}$, \\ Fahad Ibrahim Al mofeez ${ }^{4}$, Jawaher Ahmad Almusailhi ${ }^{5}$, Raseel A. Awad ${ }^{6}$ and \\ Waad Khalid Almusailhi ${ }^{6}$ \\ ${ }^{1}$ Consultant family and community medicine; moh, Saudi Arabia \\ ${ }^{2}$ General Directorate of Health Affairs in Riyadh, Saudi Arabia \\ ${ }^{3}$ MBBS, FFCM, Consultant Saudi Ministry of health \\ ${ }^{4}$ General Directorate of Health Affairs in Riyadh, Ministry of Health, Saudi Arabia \\ ${ }^{5}$ Medical student Imam Abdulrahman Alfaisal University, Dammam, KSA \\ ${ }^{6}$ Medical student Al Maarefa college, Riyadh, KSA \\ *Corresponding author
}

\section{A B S T R A C T}

The present study have been carried out to determine the Effect of Nanosilver and Antibiotics Separately and in Combination on Pseudomonas aeruginosa Clinical Isolates. There were Forty Pseudomonas aeruginosa were isolated from clinical

\section{Keywords}

Nanosilver and

Antibiotics,

Pseudomonas

aeruginosa

Article Info

Accepted:

25 September 2018

Available Online:

10 October 2018 specimens. The MicroScan WalkAway-96 SI System was used in laboratory Identification and antimicrobial susceptibility testing was performed. Also, the well diffusion method was used to evaluate the antibacterial activity of silver nanoparticles in combination with antibiotics. All isolates were susceptible to more than MIC of nanosilver compared to a high resistance to multiple classes of antibiotics. The Average of inhibition zone diameters of different concentrations of nanosilver dilutions. The combined effect of the silver nanoparticles with antibiotics was assessed in comparison to antibiotics, with well diffusion method. It was observed that the collective effect of silver nanoparticles with antibiotics was additive. The activity of the three antibiotics: gentamycine. ampicillin, and tetracycline with nanoparticles, against Pseudomonas aeruginosa was shown as percentage enhancement in antibacterial effect. Silver nano particles may provide safe and effective treatment for MDR Pseudomonas aeruginosa infections when combined with antibiotics. More further studies are required for understand synergistic effect of nanosilver combining, Assessment of safety and efficacy of new antibiotic- nanosilver combination.

\section{Introduction}

Pseudomonas aeruginosa is especially problematic for seriously ill patients in ICUs.
From 1992 to 1997, data from the National Nosocomial Infections Surveillance System showed that $P$. aeruginosa was responsible for $21 \%$ of pneumonias, $10 \%$ of urinary tract 
infections, $3 \%$ of bloodstream infections, and $13 \%$ of eye, ear, nose, and throat infections within ICUs in the United States (1). A similar study conducted in Europe identified $P$. aeruginosa as the second most frequently isolated organism in reported cases of ICUacquired infections (2). In this surveillance study, $P$. aeruginosa was accountable for $30 \%$ of pneumonias, $19 \%$ of urinary tract infections, and $10 \%$ of bloodstream infections. $P$. aeruginosa presents a serious therapeutic challenge for treatment of both communityacquired and nosocomial infections, and selection of the appropriate antibiotic to initiate therapy is essential to optimizing the clinical outcome (3, 4). Unfortunately, selection of the most appropriate antibiotic is complicated by the ability of $P$. aeruginosa to develop resistance to multiple classes of antibacterial agents, even during the course of treating an infection. Epidemiological outcome studies have shown that infections caused by drug-resistant $P$. aeruginosa are associated with significant increases in morbidity, mortality, need for surgical intervention, length of hospital stay and chronic care, and overall cost of treating the infection $(5,6,7)$. Even more problematic is the development of resistance during the course of therapy, a complication which has been shown to double the length of hospitalization and overall cost of patient care (8). P. aeruginosa can develop resistance to antibacterials either through the acquisition of resistance genes on mobile genetic elements (i.e., plasmids) or through mutational processes that alter the expression and/or function of chromosomally encoded mechanisms. Both strategies for developing drug resistance can severely limit the therapeutic options for treatment of serious infections. limit the therapeutic options for treatment of serious infections. P. aeruginosa exhibits the highest rates of resistance for the fluoroquinolones, with resistance to ciprofloxacin and levofloxacin ranging from
20 to $35 \%$. Although not surprising, the highest rates were reported for isolates obtained from patients in ICUs. P. aeruginosa isolates from ICU patients also trend toward higher rates of _-lactam resistance than general trends for hospitalized patients.

Antimicrobial properties of silver compounds had been identified for a long time $(9,10)$. In recent decades nanosilver has been welcomed because of its unique antimicrobial properties (11). Silver particles have naturally anti-inflammatory effects and also increase the power of healing $(12,13)$. It also has antimicrobial properties through effects on enzyme systems, cell wall and bacterial DNA (13). The silver particles change phosphotyrosine peptide in bacteria and subsequently change signals between the cells and ultimately prevent the proliferation of bacteria (14). However, most antimicrobial mechanisms of this material are still quite unknown (15). Maximum exposure of silver particles in the nanosilver suspension causes increased antibacterial activity of this compound. No toxic effects on human's tissues were reported reported by low concentration of nanosilver solutions $(16,17)$. Nanosilver particles are effective on the bacteria Streptococcus mutans which is one of the important pathogens in dental caries, and therefore it has anti-decay effects (18-19).

\section{Materials and Methods}

\section{Bacterial strains}

Forty Pseudomonas aeruginosa were isolated from clinical specimens at microbiology laboratory, Department of clinical analysis at King Khalid hospital, Hafr Elbatin, KSA. Mid-stream urine, suction tip, pus and blood specimens were collected aseptically for bacteriological examination. Handling, transporting and storing of collected samples were made at refrigeration temperature. All 
samples were inoculated on MacConkey's and Blood agar, incubated at $37^{\circ} \mathrm{C}$ for overnight, and colonies were processed.

\section{Micro Scan analysis}

The MicroScan WalkAway-96 SI System was used in laboratory Identification and antimicrobial susceptibility testing was performed with Neg/BP/Combo 30-B1017$306 \mathrm{E}$ combination panels. All procedures were performed according to the manufacturer's directions.

\section{Antimicrobial activity of nanosilver}

Nanosilver were obtained from SIGMA ALDRICH, SAINT LOUIS, USA. MIC was defined as the lowest concentration of nanosilver preparation that prevented bacterial growth. MICs were determined for all strains by the CLSI reference method (20). Concentrations tested were diluted: (300 ppm), (200 ppm), (100 ppm), (50 ppm), (25 ppm), (12.5 ppm), (6.25 ppm), (3.125 ppm). Disk diffusion tests were performed along with MIC determinations using one inoculum preparation.

\section{Effect of Nanosilver - Antibiotic combination}

The well diffusion method was used to evaluate the antibacterial activity of silver nanoparticles in combination with antibiotics. Based on the CLSI standard, the selected concentrations of antibiotics (HiMedia Chemicals Pvt. Ltd., Mumbai, India) were gentamycin $(10 \mu \mathrm{g})$, ampicillin $(10 \mu \mathrm{g})$, and tetracycline $(30 \mu \mathrm{g})$, Amikacine $(30 \mu \mathrm{g})$ respectively [20]. By using spread plate method, the Mueller-Hinton agar plates were inoculated with the turbidity adjusted bacterial suspension, and antibiotic with nanosilver (5 ppm) to see the combined activity placed in $6 \mathrm{~mm}$ diameter wells mad by sterile glass
Pasteur pipette.. These plates were incubated at $37^{\circ} \mathrm{C}$ for $24 \mathrm{~h}$, and zone of inhibition (ZOI) was measured by subtracting the well diameter from the total inhibition zone diameter. This assay was performed in triplicate.

\section{Results and Discussion}

The present study was examined the Pseudomonas aeurginosa growth in culture media in the presence of nanosilver compared to when the culture media contained different antibiotics; we found that nanosilver dilutions readily prevented growth of the multidrug resistant Pseudomonas aeruginosa. The information about the susceptibility and resistance rate of the isolates against different antibiotics used is shown in Figures 1,2 and against nanosilver in figure 3. This information shows the $100 \%$ resistance of Pseudomonas aeruginosa against different antibiotics such as tetracycline, cefoxitine, Maezocilline and Trimethoprim/ Sulfamethoxazole, more than $85 \%$ resistance against Cefuroxime and cefotaxime and lower resistance against piperacillin/ tazobactam $(62.5 \%)$, imipenem (50\%), and amikacin (50 $\%)$. Nanosilver examinations showed that the MIC of nanosilver was $12.5 \mathrm{ppm}$ (with average inhibition zone being $8.02 \mathrm{~mm}$ ).

Susceptibility to nanosilver depended on the concentration. All isolates were susceptible to more than MIC of nanosilver compared to a high resistance to multiple classes of antibiotics. The Average of inhibition zone diameters of different concentrations of nanosilver dilutions is shown in Figure 3. By reducing the concentration of nanosilver, its effectiveness was reduced; at about the 12.5 ppm concentration and lower, inhibition zone of bacterial growth was not observed.

The combined effect of the silver nanoparticles with antibiotics was assessed in comparison to antibiotics, with well diffusion 
method. It was observed that the collective effect of silver nanoparticles with antibiotics was additive. The activity of the three antibiotics: gentamycine. ampicillin, and tetracycline with nanoparticles, against Pseudomonas aeruginosa was shown as percentage enhancement in antibacterial effect. The increase in antibacterial activity of different antibiotics was quantified by the equation $(B-A) / A \times 100$, where $A$ and $B$ are the ZOI for antibiotic and antibiotic + silver nanoparticles, respectively. The activity of all the tested antibiotics was increased in combination with all the silver nanoparticles employed, against the test bacterial strains. The highest increase was noted for ampicillin $(19.7 \%)$ followed by gentamicin $(12.2 \%)$ and tetracycline (6.9\%) (Figure 4).

Fig.1 Overall average Antibiotic resistance percentage of Pseudomonas aeruginosa isolates against different antibiotics

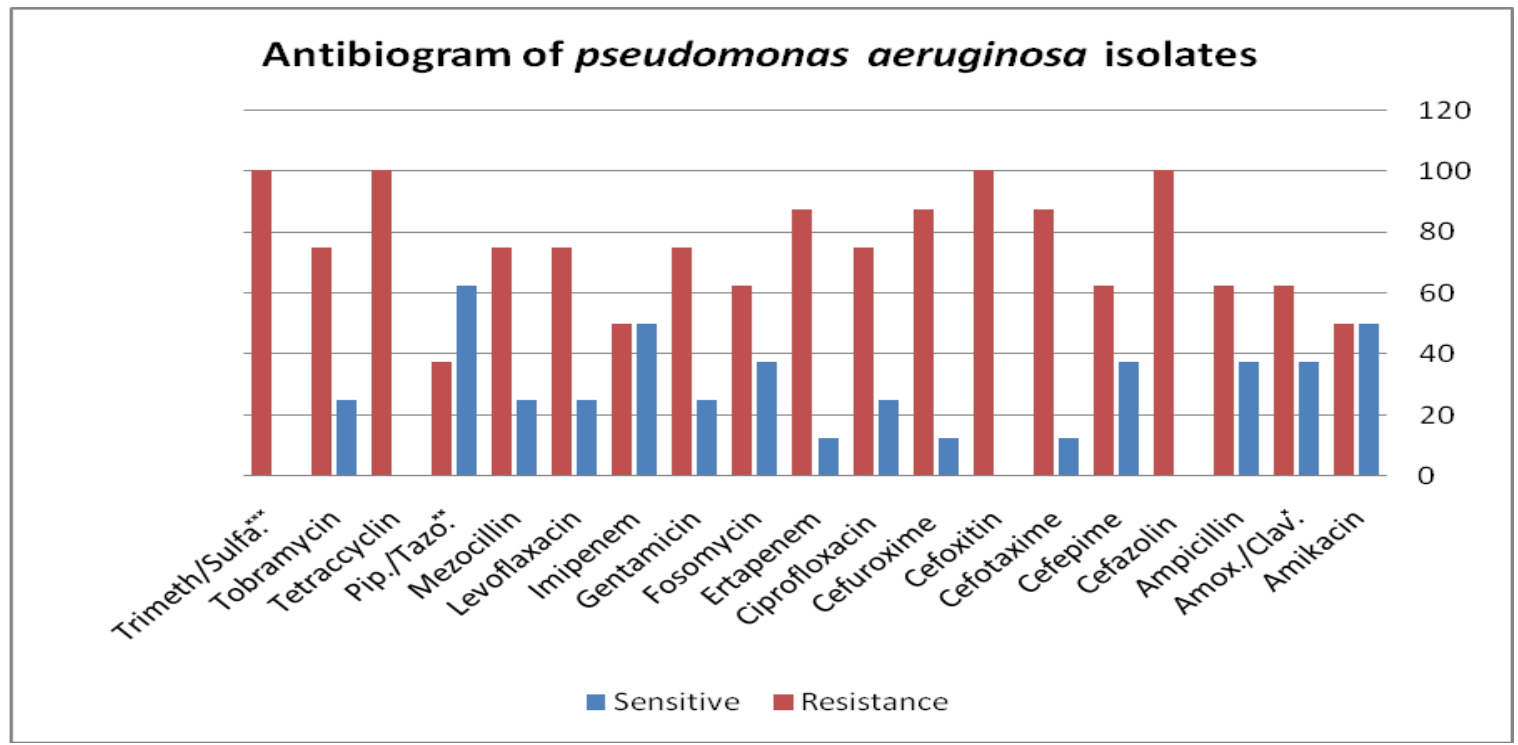

Fig.2 MIC of different antibiotics

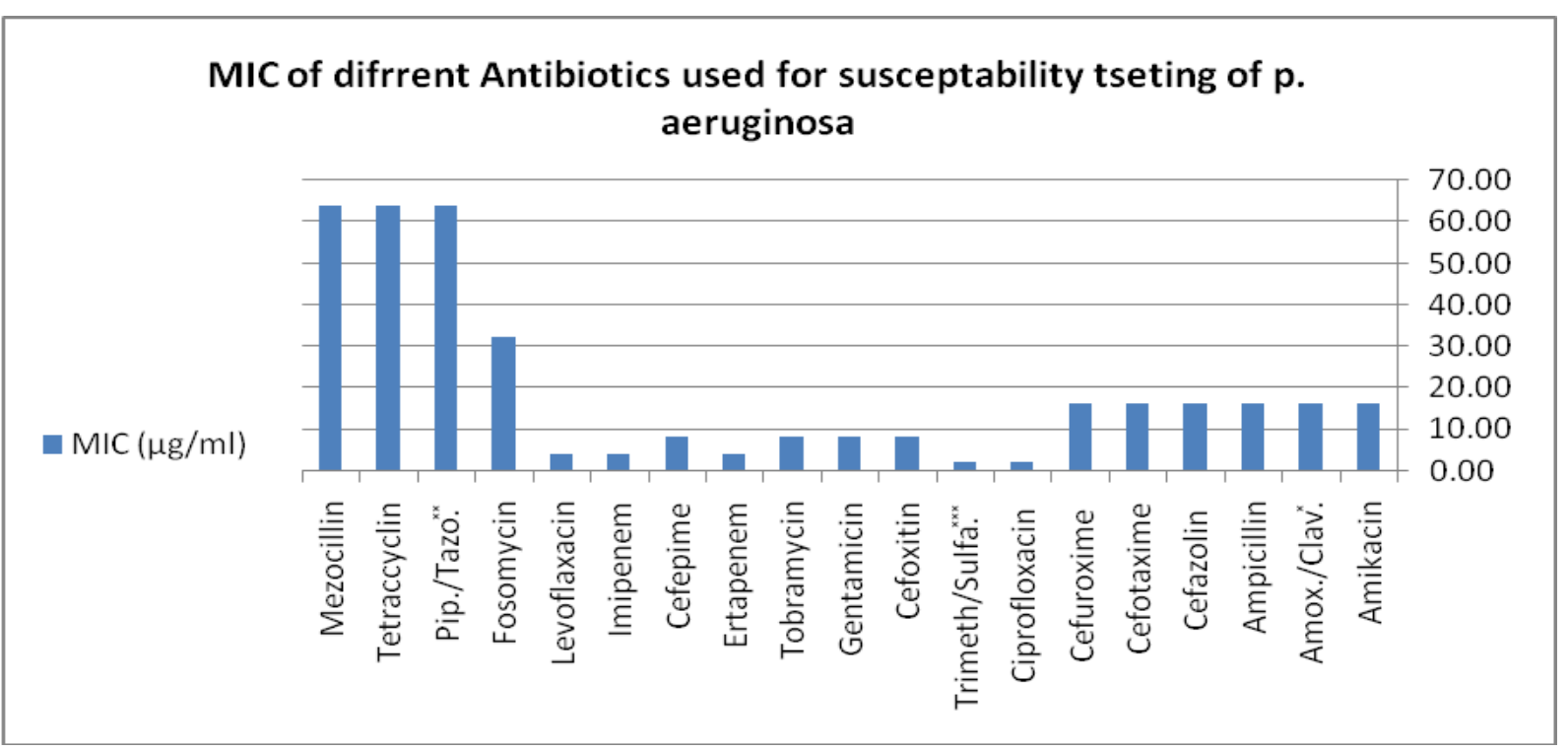


Fig.3 Antibacterial effect of different nanosilver concentrations on Pseudomonas aeruginosa isolates

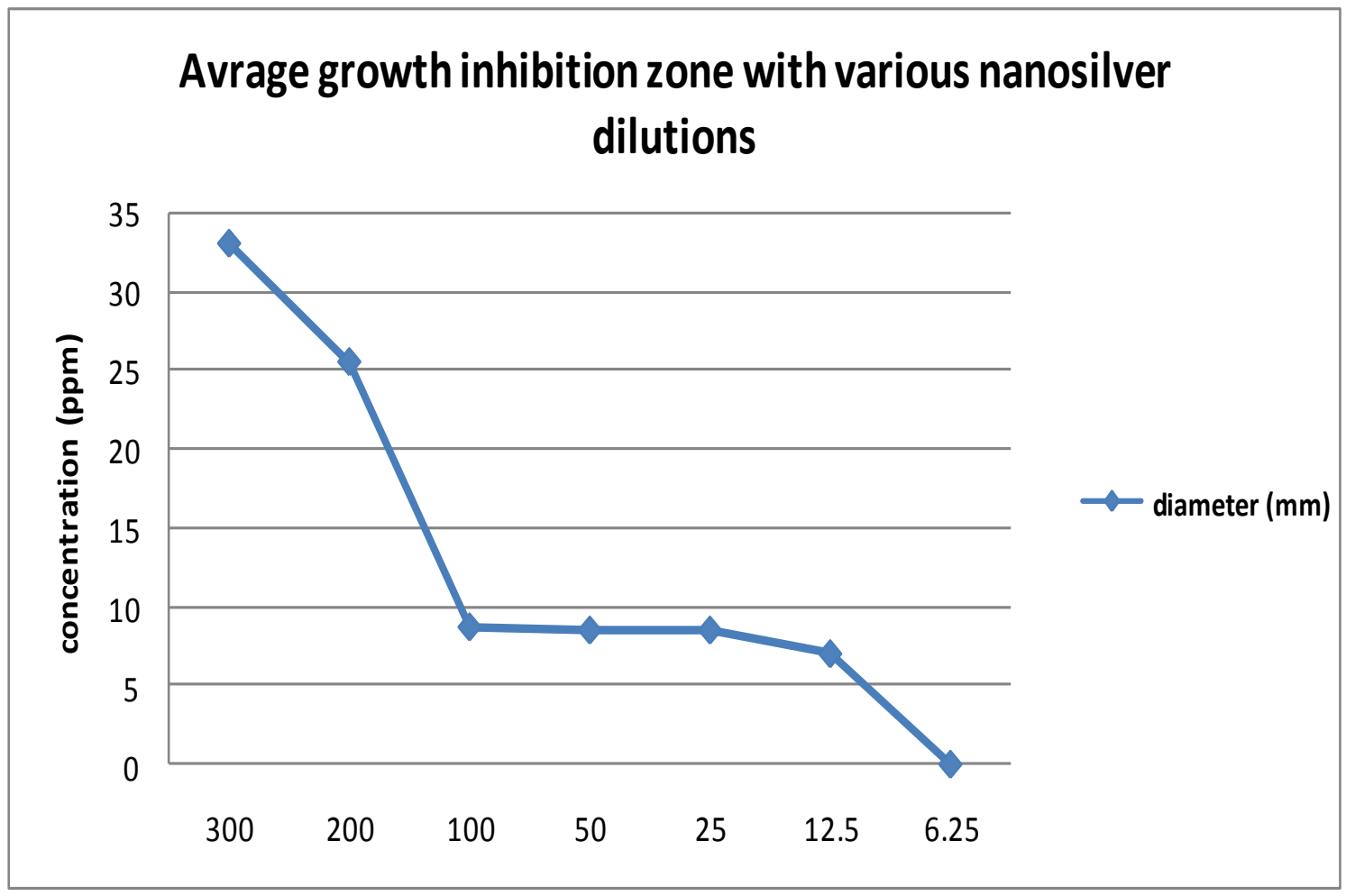

Fig.4 Effect of Antibiotic-nanosilver combination on Pseudomonas aeruginosa

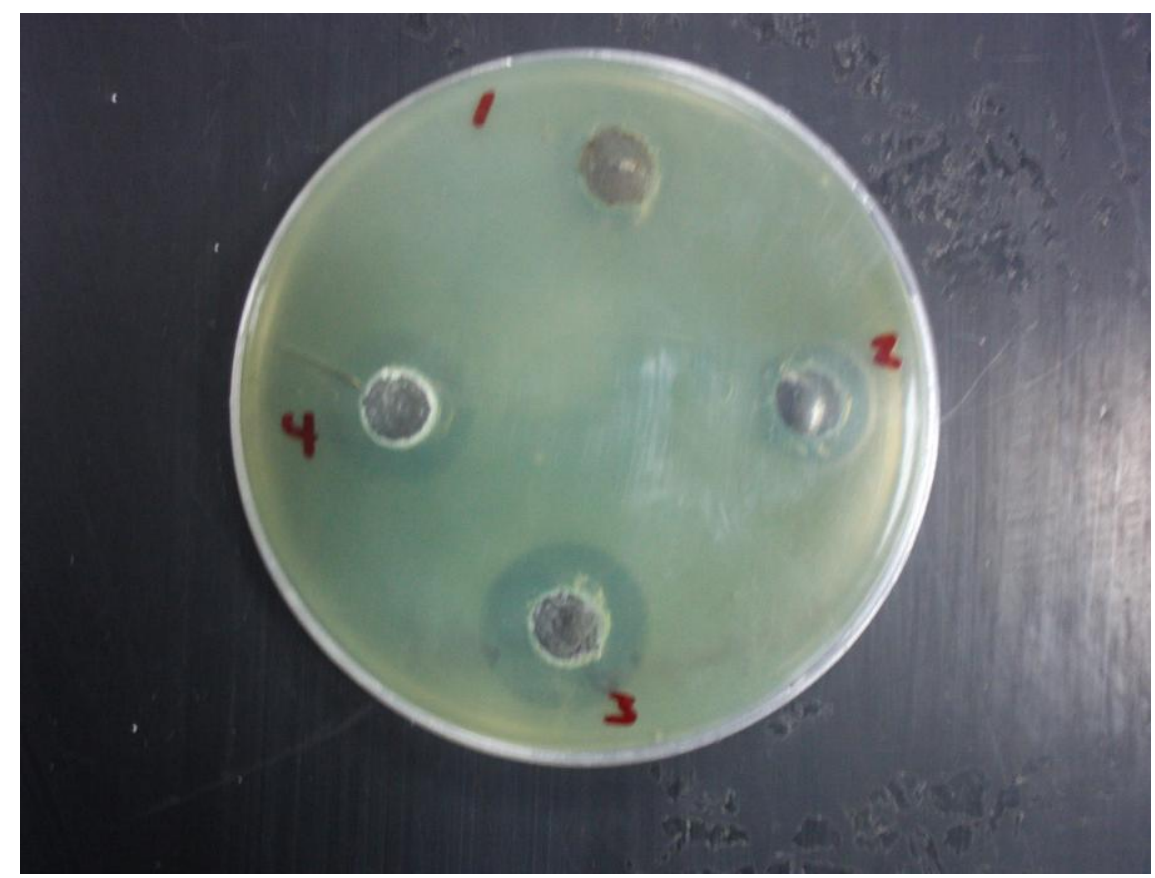

1- control, 2- nanosilver, 3- Antibiotic+ nanosilver, 4- Antibiotic 
The rising trend of resistance to antibiotics alarms about the dilemma that could be faced when treating such infections. The results showed that clinical isolates of $p$. aeruginosa in our hospitals are highly resistant to multiple classes of antibiotics. $p$. aeruginosa are important opportunistic pathogens with increasing rates of multi-antibiotic resistance due to both intrinsic and acquired mechanisms. In this study we collected 40 samples from different departments of King Khalid hospital of Saudi Arabia. Several common antibiotics against $p$. aeruginosa were used for in vitro susceptibility test. This experiment revealed high resistance rates of $p$. aeruginosa which were as follows: $100 \%$ to cefoxitine, tetracycline, cefazoline and trimethobrine/sulfamethaxazole, more than $85 \%$ resistance to Ertapenem, cefotaxime and cefuroxime, more than $60 \%$ resistance to tobramycine, Mezocelline, gentamicin, levoflaxacin and ciproflaxacin, low resistance to amikacin, piperacillin/tazobactam. Other researches in Egypt that reported high rates of antimicrobial resistance (21). In Turkey, Unan et al., reported that $60 \%$ of his $P$. aeruginosa isolates were MDR (22). In Egypt, Gad et al., observed high levels of MDR $P$. aeruginosa and that $\beta$ lactamase production is the main mechanism of resistance $(36 \%$ were MDR and $95 \%$ were ES $\beta$ Ls producer) (23), On the other hand, in Iran detected lower levels as $30 \%$ of their isolates were MDR and only 9.2\% were ES $\beta$ Ls producer (24). Our study showed high sensitivity to piprecillin/Tazobactum followed by Amikacin and Impenem, other studies showed semillar results Amikacin and imipenem were the most effective drugs against $P$. aeruginosa. The effectiveness of amikacin $(80.5 \%$ sensitive) over imipenem (66.7\% sensitive) against $P$. aeruginosa was corroborated by data from recent researches (23). Earlier studies reported that imipenem was the most effective antibiotic against $P$. aeruginosa (25). However, recent studies demonstrated the evolution of imipenem - resistant strains of $P$. aeruginosa. Carbapenems were considered to be the treatment of choice against serious ES $\beta$ Ls associated infections, however resistance to carbapenems, especially in P.aeruginosa, results from reduced levels of drug accumulation, increased expression of pump efflux or production of $\beta$-lactamases (23). Our study revealed moderate activity of ciprofloxacin $37.5 \%$. There were variable data about ciprofloxacin action Similar results about ciproflaxacin against P.aeruginosa were detected by Gad et al., (23) on the other hand Zahra (reported that ciprofloxacin exhibited high susceptibility pattern (73.6\%) (24). In our research, high resistance rates exhibited by P.aeruginosa against cefeoxitine and cefazolin $(100 \%)$. In contrast, cephalosporins tested in a study conducted in Nigeria, showed that $90 \%$ of the isolates were sensitive to it (26).

Small nanoparticles with a large surface area to volume ratio provide a more efficient means for antibacterial activity even at very low concentrations. Silver nanoparticles (Diameter 5-32 nm) enhance the antibacterial activity of various antibiotics (27). Our results showed a $100 \%$ influence by the prepared nanosilver and $12.5 \mathrm{ppm}$ MIC. Numerous researches have been performed studying the effect of nanosilver on pathogenic microorganism. Our results were quiet similar to the Humberto (27) study on Pseudomonas aeruginosa. Interestingly, among the selected antibiotics ampicillin has shown the highest activity against $p$. aeruginosa with silver anoparticles followed by gentamicin (12.2\%) and tetracycline $(6.9 \%)$. The combination effect of nanosilver and ampicillin has more potential compared to the other antibiotics and may be caused by both, the cell wall lysis action of the ampicillin and the DNA binding action of the silver nanoparticles. The probable mechanism involved in enhanced 
antibacterial activity of antibiotics with silver nanoparticles can be attributed to the bonding reaction between nanoparticles and antibiotic molecules. The active functional groups of antibiotics such as hydroxyl and amino groups react with large surface area of the silver nanoparticles by chelation (28). The mechanism of the bactericidal effect of $\mathrm{Ag}$ NPs is that they may attach to the surface of the cell membrane dis- turbing permeability and respiration functions of the cell (29). Smaller Ag NPs having the large surface area available for interaction would give more bactericidal effect than the larger Ag NPs (29). It is also possible that Ag NPs not only interact with the surface of membrane, but can also penetrate inside the bacteria (30).

The present study showed increasing of MDR Pseudomonas aeruginosa which alarms about the dilemma that could be faced when treating such infections. Silver nano particles may provide safe and effective treatment for MDR Pseudomonas aeruginosa infections when combined with antibiotics. More further studies are required for understand synergistic effect of nanosilver combining, Assessment of safety and efficacy of new antibioticnanosilver combination.

\section{References}

1. ADA Council on Dental Materials and Devices. JADA 2008. p. 673-7.

2. Aloush, V., S. Navon-Venezia, Y. Seigman-Igra, S. Cabili, and Y. Carmeli. 2006. Multidrug-resistant Pseudomonas aeruginosa: risk factors and clinical impact. Antimicrob. Agents Chemother. 50:43-48.

3. Bisbe, J., J. M. Gatell, and J. Puig. 1988. Pseudomonas aeruginosa bacteremia: univariate and multivariate analyses of factors influencing the prognosis in 133 episodes. Rev. Infect. Dis. 10:629-635.
4. Brown TA, Smith DG. 1976. The effects of silver nitrate on the growth and ultrastructure of the yeast Cryptococcusus albidus. Microbios Lett. 3:155-62.

5. Carmeli, Y., N. Troillet, A. W. Karchmer, and M. H. Samore. 1999. Health and economic outcomes of antibiotic resistant Pseudomonas aeruginosa. Arch. Intern. Med. 159:1127-1132.

6. Caufield PW, Dasanayake AP, Li Y, Pan Y, Hsu J, Hardin JM. 2000. Natural history of Streptococcus sanguinis in the oral cavity of infants: evidence for a discrete window of infectivity. Infect Immun.68:4018-23.

7. Chaloupka K, Malam Y, Seifalian AM. 2010. Nanosilver as a new generation of nanoproduct in biomedical applications. Trends Biotechnol. 28:580-8.

8. Dimatatac, E. L., M. M. Alejandria, C. Montalban, C. Pineda, C. Ang, and R. Delino. 2003. Clinical outcomes and costs of care of antibiotic resistant Pseudomonas aeruginosa infections. Philipp. J. Microbiol. Infect. Dis. 32:159167.

9. El-Kholy, A., Baseem, H., Hall, G., Procop, G. \& Longwoth, D. (2003). "Antimicrobial Resistance in Cairo, Egypt, 1999-2000: Survey of 5 Hospitals," Journal of Antimicrobial Chemotherapy. 51: 625-630.

10. Fayaz, A. M., K. Balaji, M. Girilal, R. Yadav, P. T. Kalaichelvan, and R. Venketesan. (2010). Biogenic synthesis of silver nanoparticles and their synergistic effect with antibiotics: a study against gram-positive and gram-negative bacteria.

Nanomedicine: Nanotechnology, Biology, and Medicine, 6:103-109.

11. Gad, G. F., El- Domany, R. A., Zaki, S. \& Ashour, H. M. (2007). "Characterization of Pseudomonas Aeruginosa Isolated from Clinical and 
Environmental Samples in Minia, Egypt: Prevalence, Antibiogram and Resistance Mechanisms," Journal of Antimicrobial Chemotherapy.60: 1010-1017.

12. Gales, A. C., Sader, H. S. \& Jones, R. N. (2002). "Urinary Tract Infection Trends in Latin American Hospitals: Report from the SENTRY Antimicrobial Surveillance Program (1997-2000)," Diagn Microbiol Infect Dis. 44: 289-99.

13. Gasink, L. B., N. O. Fishman, M. G. Weiner, I. Nachamkin, W. B. Bilker and E. Lautenbach. 2006. Fluoroquinoloneresistant Pseudomonas aeruginosa: assessment of risk factors and clinical impact. Am. J. Med. 119: 526e19$526 \mathrm{e} 25$.

14. Hernandez-Sierra JF, Ruiz F, Pena DC, Martinez-Gutierrez F, Martinez AE, Guillen Ade J, et al., 2008. The antimicrobial sensitivity of Streptococcus mutans to nanoparticles of silver, zinc oxide, and gold. Nanomedicine. 4:23740.

15. Jo YK, Kim BH, Jung G. 2009. Antifungal activity of silver ions and nanoparticles on phytopathogenic fungi. Plant Dis. 93:1037-43.

16. Jung WK, Koo HC, Kim KW, Shin S, Kim SH, Park YH. 2008. Antibacterial activity and mechanism of action of the silver ion in Staphylococcus aureus and Escherichia coli. Appl Environ Microbiol.74:2171-8.

17. Klasen HJ. 2000. Historical review of the use of silver in the treatment of burns. I. Early uses. Burns.26:117-130.

18. Kvitek, L., A. Panacek, J. Soukupova, M. Kolar, R. Ve-cerova, R. Prucek, et al., (2008).Effect of Surfactants and Polymers on Stability and Antibacterial Activity of Silver Nanoparticles (NPs)," Journal of Physical Chemistry C.112:5825-5834.

19. Lara Humberto H, Ayala-Núnez Nilda V, Ixtepan Turrent Liliana del Carmen,
Rodríguez Padilla Cristina. (2010) Bactericidal effect of silver nanoparticles against multidrug-resistant bacteria. World J Microbiol Biotechnol.26:615621.

20. Micek, S. T., A. E. Lloyd, D. J. Ritchie, R. M. Reichley, V. J. Fraser, and M. H. Kollef. 2005. Pseudomonas aeruginosa bloodstream infection: importance of appropriate initial antimicrobial treatment. Antimicrob. Agents Chemother. 49:1306-1311.

21. Morones, J. R., J. L. Elechiguerra, A. Camacho, K. Holt, J. Kouri, J. T. Ramirez, et al., (2005) The Bactericidal Effect of Silver Nanoparticles.Nanotechnology.16:2346.

22. Oni, A. A., Nwaorgu, O. G. B., Bakare, R. A., Ogunkunle, M. O. \& Toki, R. A. (2002). 'The Discharging Ears in Adults in Ibadan, Nigeria; Causative Agents and Antimicrobial Sensitivity Pattern,' Afr J Clin Exp Microbiol.3:3-5.

23. Panyala NR, Peña-Méndez EM, Havel J. 2008. Silver or silver nanoparticles: a hazardous threat to the environment and human health. J Appl Biomed.6:117-29.

24. Richards, M. J., J. R. Edwards, D. H. Culver, R. P. Gaynes, et al., 1999. Nosocomial infections in medical intensive care units in the United States. Crit. Care Med. 5:887-892.

25. Richards, M. J., J. R. Edwards, D. H. Culver, R. P. Gaynes, et al., 1999. Nosocomial infections in pediatric intensive care units in the United States. Pediatrics 103:e39.

26. Safavi K, Esfahanizadeh M, Mortazaeinezahad DH, Dastjerd H. 2011. The study of nano silver (NS) antimicrobial activity and evaluation of using NS in tissue culture media. Etlibrary.3:159-61.

27. Shahverdi AR, Fakhimi A, Shahverdi HR, Minaian S. (2007) Synthesis and effect of silver nanoparticles on the 
antibacterial activity of different antibiotics against Staphylococcus aureus and Escherichia coli. Nanomedicine.3:168-71.

28. Shrivastava S, Bera T, Singh SK, Singh G, Ramachandrarao P, Dash D. 2009. Characterization of antiplatelet properties of silver nanoparticles. ACS Nano.3:1357-64.

29. Slawson RM, Van Dyke MI, Lee H, Trevors JT. 1992. Germanium and silver resistance, accumulation, and toxicity in microorganisms. Plasmid. 27:72-9.

30. Unan, D., Gnseren, F. et al., (2000). 'The Resistance of $\mathrm{P}$. aeruginosa Strains
Isolated from Nosocomial Infections against Various Antibiotics,' Mikrobiyol Bult.34: 255-60.

31. Watts, J. L., 2008 . Performance Standards for Antimicrobial Disk and Dilution Susceptibility Tests for Bacteria Isolated from Animals: Approved Standard, Clinical and Laboratory Standards Institute, 3rd edition.

32. Zahra, T. \& Moniri, R. (2011). "Detection of ESBLs and MDR in Pseudomonas Aeruginosa in a TertiaryCare Teaching Hospital," Iranian Journal of Clinical Infectious Diseases. 6:18-23.

\section{How to cite this article:}

Alyousef mansour Ali, Abdulaziz Ibrahim Alsenaid, Ahmed Hassan Al-Mosilhi, Fahad Ibrahim Al mofeez, Jawaher Ahmad Almusailhi, Raseel A. Awad and Waad Khalid Almusailhi. 2018. Effect of Nanosilver and Antibiotics Separately and in Combination on Pseudomonas aeruginosa Clinical Isolates. Int.J.Curr.Microbiol.App.Sci. 7(10): 3695-3703. doi: https://doi.org/10.20546/ijcmas.2018.710.427 